\title{
Konsequente Umsetzung von Zielen durch Coaching
}

\author{
Praktisch nützliche Erkenntnisse aus der Grundlagenforschung und ihre Anwendung
}

\author{
Siegfried Greif • Elke Benning-Rohnke
}

Online publiziert: 14. Juli 2015

(C) Die Autor(en) 2015. Dieser Artikel ist auf Springerlink.com mit Open Access verfügbar.

Zusammenfassung Handlungsziele werden oft nicht praktisch umgesetzt. Erkenntnisse und Erfahrungen legen nahe, dass die unzureichende Formulierung konkreter Umsetzungsabsichten sowie die fehlende Begleitung in der Verstetigung neuer Verhaltensroutinen dafür maßgeblich sind. Der vorliegende Beitrag vermittelt wissenschaftliche Erkenntnisse aus der Grundlagenforschung und stellt Coaching-Interventionen zur Unterstützung der Umsetzung in die Praxis vor. Anhand von zwei Praxisprojekten in Banken wird beispielhaft gezeigt, wie ein stringentes Vorgehen in Anlehnung an die Methode des Ergebnisorientierten Coachings durchgeführt werden kann, mit Unterstützung der Formulierung konkreter Handlungsziele im Team, Entwicklung individueller Handlungsabsichten, Umsetzungsunterstützung und Verstetigung zur nachhaltigen Verhaltensveränderung. Nach einer Panelerhebung hat sich in einem der Projekte die Zielerreichung in Profitabilität um $29 \%$ verbessert und in Wachstums sogar um 105\%. Abschließend wird empfohlen, Erkenntnisse und Methoden aus weiteren Feldern der Grundlagenforschung zur Innovation des Change Managements und Coachings in Unternehmen und Coachingpraxis zu nutzen.

\footnotetext{
S. Greif $(\bowtie)$

Institut für Psychologie (i. R.), Universität Osnabrück und Institut für wirtschaftspsychologische Forschung und Beratung (IwFB)

$\mathrm{GmbH}$,

Osnabrück, Deutschland

E-Mail: sgreif@uos.de

E. Benning-Rohnke

zeb,

München, Deutschland

E-Mail: ebenning@zeb.de
}

Schlüsselwörter Rubikonmodell · Shadowing · Transfercoaching $\cdot$ Umsetzungsabsichten $\cdot$ Volition

\section{Rigorous Implementation of Goals Through Coaching}

Practically Beneficial Findings from Basic Research and Their Application

\begin{abstract}
Action goals are often not put into practice. Based on findings and experiences, this is due to poor formulation of specific implementation intentions, and also the missing support for the habituation of new behavior routines. The contribution reveals scientific findings from basic research and presents coaching interventions, which support the transfer of action goals into practice. Two change projects in banks demonstrate how a stringent procedure can be applied, based on the methods of resultoriented coaching, together with methods, which support the formulation of specific action goals, individual implementation intentions, rigorous transfer into practice and continuation. Results of a panel survey show that in one of the projects, the achievement of goals improved by $29 \%$ in profitability and $105 \%$ in growth. Finally, the authors recommend that findings and methods from further fields of basic research be used for innovation of change management and coaching practice in companies.
\end{abstract}

Keywords Rubicon-model $\cdot$ Shadowing $\cdot$ Transfer coaching $\cdot$ Implementation intentions $\cdot$ Volition 


\section{Warum ist es so schwer Ziele umzusetzen?}

\subsection{Die Kluft zwischen Zielen und Handlungen}

Wer kennt das nicht: Wir nehmen uns etwas vor, aber setzen es nicht um. Warum tun wir nicht, was wir uns vorgenommen haben? Zwischen Zielen und Handlungen besteht anscheinend eine Kluft, die so genannte Action Intention Gap. Die Frage, wie sie überbrückt werden kann, ist eine der großen herausfordernden Fragestellungen der anwendungsorientierten psychologischen Forschung. Im Beitrag wird einleitend das Rubikonmodell vorgestellt, das eine Antwort auf diese Frage gibt. Wie ausgeführt wird, reicht es nach diesem Modell nicht, wenn wir uns Ziele setzen. Um die Wahrscheinlichkeit zu erhöhen, dass unsere Ziele in Handlungen umgesetzt werden, sind zusätzlich bewusste Willensentscheidungen mit genau formulierten Umsetzungsabsichten erforderlich. Das Modell mit den daraus abgeleiteten, empirisch belegten Interventionsmethoden ist für die Anwendung im Coaching beachtenswert, weil es die im Coaching gebräuchlichen Methoden der Zielklärung ergänzt. Als wissenschaftlich fundiertes allgemeines Grundlagemodell ist es interessant, weil es die allgemeinen psychologischen Phasen beschreibt, die durchlaufen werden müssen, damit aus Zielen praktische Handlungen folgen.

Das Rubikonmodell schließt die Kluft zwischen Intention und Handeln allerdings nicht vollständig. Außer Betracht bleiben externe Umsetzungsbarrieren (z. B. Änderungswiderstände im sozialen Umfeld) oder innere psychische Umsetzungshemmnisse (z. B. schwer zu durchbrechende Verhaltensgewohnheiten). Im Beitrag werden Erweiterungen und Ergänzungen zum Rubikonmodell vorgeschlagen, die darauf näher eingehen und die Planung der Überwindung der Barrieren und Hemmnisse sowie ihre Bewältigung in der Umsetzungsphase in den Mittelpunkt stellen. Als Coachingmethoden zur Begleitung und Förderung der Umsetzung in dieser Phase werden zwei Methoden vorgestellt: 1. das Virtuelle Transfercoaching (VTC) von Geißler (2011) und 2. das Telefon-Shadowing (Greif 2013).

Anschließend werden Erfahrungen und Ergebnisse aus zwei Veränderungsprojekten berichtet, in denen unter Berücksichtigung des Rubikonmodells gemeinsame und individuelle Ziele sowie Umsetzungsabsichten gefördert wurden und in der Umsetzung die Methode des Virtuellen Transfercoachings und in Einzelfällen ergänzend das Telefon-Shadowing eingesetzt wurde. In der abschließenden Diskussion werden Perspektiven der Kooperation von Wissenschaft und Praxis zur Integration von Erkenntnissen und Methoden aus der Grundlagenforschung bei der Überbrückung der Kluft zwischen Zielen und Handlungen diskutiert.
1.2 Das Rubikonmodell

\subsubsection{Zielklärung nach SMART-Kriterien}

Eine erste Antwort auf die Frage, warum Ziele nicht umgesetzt werden, gibt die in zahlreichen Untersuchungen überprüfte Zielsetzungstheorie von Locke und Latham (2002). Ihre Erkenntnisse gehören heute zum Allgemeinwissen im Feld der Personalentwicklung. Um die Umsetzungsmotivation zu fördern, sollen Ziele herausfordernd sein und nach den SMART-Kriterien definiert werden: spezifisch, messbar, erreichbar (bzw. attainable), realistisch und zeitlich festgelegt, (bzw. time-bound) (Doran 1981). Auch im Coaching zählen diese Kriterien seit langem zu den wissenschaftlichen Grundlagen der Zielklärung (David et al. 2013). Allerdings überschätzen Anwender(innen) dieser probaten Kriterien oft die Wahrscheinlichkeit, mit der klare Zielintentionen später in Verhalten umgesetzt werden. Nach einer Auswertung von zehn Metaanalysen mit insgesamt 422 Studien und 82.107 Untersuchungsteilnehmer(inne)n werden Zielintentionen später im Durchschnitt lediglich von $28 \%$ ausgeführt (Sheeran 2002). Im Coaching mag dieser Prozentsatz höher liegen, weil das persönliche Commitment gegenüber dem Coach die Umsetzung fördern kann. Allerdings zeigen praktische Beobachtungen gleichzeitig auch, dass Klient(inn)en ihre Ziele von Sitzung zu Sitzung immer wieder ändern ohne sie konsequent weiter zu verfolgen.

\subsubsection{Von der Zielintention zur Umsetzungsabsicht - das Rubikonmodell}

Das Rubikonmodell verspricht, dass mit einer methodischen Ergänzung der Zielklärung höhere Umsetzungsraten erreicht werden können (Gollwitzer et al. 1996). Danach ist eine „Zielintention“ nur ein erster vorbereitender Schritt für die individuelle Entscheidung und Festlegung der Person auf eine bestimmte geplante Handlung. Das Ziel führt erst dann zur praktischen Handlung, wenn die Person in einer Phase der Abwägung verschiedener Ziele und Handlungen in einem zusätzlichen Akt eine feste „Umsetzungsabsicht“ (engl. implementation intention) formuliert und damit einen bewussten willentlichen Entschluss trifft (eine so genannte Volition).

Heckhausen (1991) unterscheidet im Rubikonmodell vier Handlungsphasen (vereinfacht zusammengefasst):

A. Prädezisionale motivationale Phase (Abwägen der motivationalen Werte verschiedener Ziele und der subjektiven Wahrscheinlichkeiten, sie zu erreichen; Auswahl eines bevorzugten Ziels)

B. Präaktionale volitionale Phase (Planung der Handlungen, durch die das Ziel erreicht werden kann; bewuss- 
ter willentlicher Entschluss, die geplante Handlung auszuführen)

C. Handlungsphase (willentlich gesteuerte Umsetzung der beabsichtigten Handlung unter flexibler Berücksichtigung der situativen Bedingungen)

D. Postaktionale Evaluation (Soll-Ist-Vergleich der Umsetzung der Handlungen und Zielerreichung; ggf. erneutes Abwägen veränderter oder neuer Ziele)

Die Bezeichnung „Rubikonmodell“ bezieht sich metaphorisch auf die Überschreitung des kleinen oberitalienischen Flusses Rubikon durch Julius Cäsar mit seinen Legionen am 10. Januar im Jahr 49 v. Chr. und den berühmten Ausspruch „Alea iacta est“ (der Würfel ist gefallen), den er zuvor getan haben soll. Der Fluss war eine eherne Grenze, die, um Rom und die Republik gegen militärische Machtübernahme zu schützen, von keinem Heerführer mit seinen Legionären überschritten werden durfte. Wer dieses Gesetz übertrat, war unmittelbar zum Tode verurteilt. Cäsars Spruch gilt deshalb seither als Prototyp für die Formulierung einer festen Willensentscheidung mit einem ,,point of no return“.

Das Rubikon-Phasenmodell bezieht sich keineswegs nur auf große strategische Willensentschlüsse und Handlungen, sondern auf alle Arten menschlicher Umsetzungsabsichten und Handlungen. Der Verweis auf das historische Beispiel ist lediglich metaphorisch gemeint. Betont wird damit die besondere Bedeutung des Akts der bewussten Willensentscheidung (bzw. Volition), mit der sich eine Person vor dem Handeln auf eine bestimmte Handlung und Umsetzungsabsicht festlegt.

\subsubsection{Methoden zur Formulierung von Umsetzungsabsichten}

In den gängigen Untersuchungen wird die Formulierung von Umsetzungsabsichten mit einer sehr einfachen Methode gefördert (Gollwitzer und Sheeran 2006). Die Teilnehmenden werden gebeten, zu überlegen, welches Verhalten sie zur Zielerreichung umsetzen wollen und anschließend aufgefordert ganz genau auszuformulieren, in welcher Situation und nach welchen Hinweisreizen (sogenannten „Cues") sie das beabsichtige Verhalten künftig durchführen wollen. Eine typische Formulierung hat die Form eines Wenndann-Satzes. Ein Beispiel aus einer Untersuchung: „Wenn jemand in einer Gruppendiskussion eine fremdenfeindliche Äußerung abgibt, dann beteilige ich mich mit einer Gegenstellungnahme." In der Veränderungsbegleitung in zwei Projekten im Bankenbereich wurde den Teilnehmer(inne)n die Bedeutung des individuellen Willensentschlusses, wie unten näher beschrieben, als Stufe auf einer „Erfolgstreppe“ vermittelt.

Im Einzelcoaching kann das Commitment beim Willensentschluss mit Formulierungen intensiviert werden, wobei die Klient(inn)en aufgefordert werden, sich ganz bewusst etwas Zeit dafür zu nehmen, einen ganz festen Entschluss $\mathrm{zu}$ fassen, sich vorzustellen wie sie in der Situation ihren Willen stark machen und ihre Kraft aufbauen und ihren Entschluss umsetzen. Den Moment, in dem sich die Klient(inn) en zur Umsetzung entschließen, kann man im Coaching oft daran erkennen, dass sie ihre Körperhaltung straffen und mit fester Stimme sprechen.

\subsubsection{Empirische Untersuchungen zu den Effekten von Umsetzungsabsichten}

In einer Meta-Analyse von 94 Studien haben Gollwitzer und Sheeran (2006) die Effekte von Zielintentionen ohne und mit Umsetzungsabsichten verglichen. Wenn in den Untersuchungen die Formulierung von Umsetzungsabsichten gefördert wurde, erhöht dies die Umsetzung bei zahlreichen angestrebten Zielen, z. B. akademischen, personalen und gesundheitlichen Zielen. Der zusätzliche Effekt zu Zielintentionen ist statistisch signifikant (5\% Irrtumswahrscheinlichkeit) und mittelmäßig bis stark (im Durchschnitt $\mathrm{d}=0,65)$. Nach diesen ermutigenden Ergebnissen wurden diese Methoden zur Aktivierung von Umsetzungsabsichten in praktische Konzepte zur Umsetzung von Gesundheitsverhalten aufgenommen (Schwarzer 2004, S. 93 ff.)

\subsection{Erweitertes Rubikonmodell}

\subsubsection{Nicht vorhergesehene Schwierigkeiten in der Umsetzung}

Die Autoren des Rubikonmodells bezweifeln nicht, dass Kontextbedingungen und Personenmerkmale die Umsetzung der Handlungsabsichten und die danach erwartete Zielerreichung verhindern können, berücksichtigen sie aber nicht systematisch in ihrem Modell. Im Grunde zeigte es sich bereits im historischen Beispiel Cäsars, dass er das angestrebte Ziel keineswegs allein mit seinem Willensentschluss und durch die Überschreitung des Rubikons und seinen Einmarsch in Rom erreichen konnte. So hatte er nicht damit gerechnet, dass sein Opponent, der Konsul Gnäus Pompeius Magnus, die Stadt Rom nicht verteidigte, sondern mit dem Senat und vielen Anhängern verlassen würde und dabei viele von Cäsar benötigte Güter mitnehmen konnte. In der Folgezeit musste Cäsar Geld für die versprochene Honorierung seiner Legionäre beschaffen und Unterstützer gewinnen. Erst nach mehreren Kriegen und seinem Sieg in der Schlacht von Pharsalus am 9. August im Jahr 48 v. Chr. über die zahlenmäßig weit überlegenen Legionen von Pompeius konnte er sein Ziel erreichen und die Macht über das römische Reich gewinnen.

Wie das historische Beispiel zeigt, musste Cäsar nach der Rubikonüberschreitung schwierige Situationen bewältigen, 
in denen seine Handlungen nicht zu den erhofften Ergebnissen führten. Er musste dabei jeweils das Verhalten seiner Gegner und die Folgen seiner Handlungen beobachten, ergebnisorientiert analysieren und reflektieren, sich neue Zwischenziele setzen, Entscheidungen über Handlungsabsichten treffen und ihre Umsetzung planen und durchführen. Solche Handlungszyklen, in denen nach umgesetzten Plänen unerwartete Folgen auftreten, was wiederum einen erneuten Zyklus der Beobachtung, ergebnisorientierte Reflexion, Zielklärung, Umsetzungsentscheidung und Planung erforderlich macht, lassen sich fachlich als Ungewissheitsmanagement einordnen. Derartige Zyklen sind im Allgemeinen für das Change Management komplexer organisationaler Veränderungsprozesse charakteristisch (Greif et al. 2004). Auch bei weniger komplexen Veränderungen kann durch unerwartete Handlungsfolgen ein derartiges Ungewissheitsmanagement erforderlich sein. So gibt es in den Untersuchungen über die Effekte von Umsetzungsabsichten (Gollwitzer und Sheeran 2006) Hinweise darauf, dass die Effekte geringer werden, wenn die Handlungsfolgen für die Person nicht vorhersehbar sind. Im Rubikonmodell wäre demnach zu ergänzen, dass in ungewissen Umsetzungssituationen zur Zielerreichung zusätzliche Beobachtungs-Reflexions-Zielklärungs-Umsetzungsabsichten-Handlungs-Zyklen erforderlich sind, um die Situation erfolgreich zu bewältigen. Nach vorliegenden Theorien und Forschungsergebnissen sind beim Ungewissheitsmanagement zwei Maxime Erfolg versprechend (zusammenfassend Greif 2013): 1. „Erwarte das Unerwartete“ und 2. „Registriere und analysiere kontinuierlich kleine Hinweise und Veränderungen". Sie können sowohl für das Change Management auf der organisationalen Ebene, als auch beim Einzelcoaching als Orientierungshilfe dienen.

\subsubsection{Verändern von Gewohnheiten}

Wenn starke Verhaltensgewohnheiten geändert werden sollen, erschwert das die Umsetzung von Handlungsabsichten (Adriaanse et al. 2011). Erkenntnisse der neueren Forschung zur Veränderung des Gesundheitsverhaltens nach Erkrankungen belegen besonders eindrucksvoll wie schwer es ist, Gewohnheiten zu verändern. Ein Beispiel sind Patient(inn) en, denen nach Herz-Kreislauferkrankungen von ihrem Arzt nach der Rehabilitation dringend angeraten wird, sich künftig regelmäßig sportlich zu bewegen und gesünder zu ernähren. Sie sehen die Notwendigkeit ein und nehmen sich fest vor, diese Empfehlungen konsequent umzusetzen. Nach den Ergebnissen einer einschlägigen Studie halten sie die Umsetzung ihrer Handlungsabsicht allerdings im Durchschnitt nicht mehr als drei Monate durch (Tierney et al. 2011).

Lally et al. (2010) haben untersucht, wie lange es dauert, bis Studierende eine neue stabil automatisierte Gewohnheit entwickeln. Die Untersuchungsteilnehmer(innen) konnten selbst wählen, was sie künftig täglich machen wollen (z. B. beim Mittagessen eine Flasche Wasser trinken oder vor dem Essen $15 \mathrm{~min}$ zu joggen). Im Durchschnitt waren tägliche Wiederholungen über einen Zeitraum von 66 Tagen erforderlich, damit sich eine neue automatisierte Verhaltensgewohnheit mit konsequenter Umsetzung an mehr als $95 \%$ der Tage herausbildete. Der Range, den einzelne Personen benötigen, war mit 18 bis 254 Tagen sehr groß.

Auch in anderen Verhaltensfeldern wird der Veränderung von Gewohnheiten eine hohe Bedeutung zugemessen. In der Unternehmensbefragung von Capgemini (2012) zum Change Management meinen 63,8\% der Befragten, dass „erst einmal alte Gewohnheiten überwunden werden (müssen), um Veränderungen zu erreichen“.

In seiner Darstellung aktueller wissenschaftlicher und praktischer Erkenntnisse über menschliche Gewohnheiten folgert der Wissenschaftsjournalist Duhigg (2012), dass Gewohnheiten unser tägliches Handeln stärker beeinflussen als bewusste Entscheidungen und einen enormen Einfluss auf Gesundheit, Leistung, finanzielle Sicherheit und Glück haben. Gewohnheiten basieren auf einer durch häufige Wiederholungen gefestigten zyklischen Verkoppelung von Handlungen und erwarteten Ergebnissen bzw. Auslösereizen (Cues oder Trigger) mit Verhaltensroutinen, die belohnend sind. Wenn diese Routinen erlernt wurden, werden sie automatisch unter Beteiligung der Basalganglien aktiviert, ohne dass dabei kortikale Prozesse erforderlich sind (Yin und Knowlton 2006). Sie werden gewissermaßen ohne intellektuelle Anstrengung ausgeführt und die basale Aktvierung dieser Prozesse kann auch nicht bewusst wahrgenommen werden. Die Tendenz ist stark, dass sich die Gewohnheit immer wieder unwillentlich durchsetzt, wenn die Auslöser zugegen sind. Das erklärt auch, warum kleine Ablenkungen genügen, um zu „vergessen“, dass man eine Gewohnheit ändern wollte oder dass die beabsichtigten Verhaltensänderungen in Stresssituationen nur sehr schwer umgesetzt werden können. Um eine feste Gewohnheit zu verändern, muss nicht nur die Routine, sondern möglichst zuvorderst der automatische Auslöser verändert werden. Es erfordert einen langen Lernprozess, in dem neue oder von der Person anders wahrgenommene Auslöser mit Routinen verkoppelt werden, die unmittelbar belohnend sind. Wenn beispielsweise der gemütliche Sessel mit dem daneben liegenden Buch durch ein Paar Sportschuhe (oder einem Bild der Schuhe auf dem Buch) ersetzt wird, kann dieser neue Hinweisreiz helfen, als neue Gewohnheit Walking statt Ausruhen einzuüben (Orbell und Verplanken 2010). Im Coaching hat es sich bewährt, mit Klient(inn)en, die sich vorgenommen haben, sich in Besprechungen anders als bisher zu verhalten, als Erinnerungshilfe oben auf ihren Notizblock ein nur für sie verständliches Symbol zu zeichnen oder ein Post-it zu verwenden. Auf Coachingmethoden zur 
Unterstützung der Umsetzung über einen längeren Lernzeitraum wird unten zurückgekommen.

Nicht nur durch die individuellen Gewohnheiten der Person, die sich ändern will, erschweren die Umsetzung von Handlungsabsichten. Auch das soziale Umfeld mit seinen Erwartungen und Gewohnheiten kann die Veränderung verhindern. In jedem Veränderungsprozess in einem Unternehmen sind Änderungswiderstände der Beteiligten die täglichen Begleiter der Führungskräfte und Mitarbeiter(inn) en. Unbearbeitet können sie Misserfolgserlebnisse, generalisierte Frustration und Veränderungsmüdigkeit erzeugen. Wenn die Person den Eindruck hat, dass sie nicht umsetzen kann, was sie unbedingt tun wollte, weil sie von anderen daran gehindert wird, wirkt das stark demotivierend. Eine „Remotivierung“ ist schwerer als vor dieser frustrierenden Erfahrung.

\subsubsection{Vorbereitung und Unterstützung der Umsetzung im erweiterten Rubikonmodell}

Um die angesprochenen Schwierigkeiten und Lösungsansätze zu berücksichtigen, werden im Folgenden im Rubikonmodell Vorbereitungen und Unterstützungen der Umsetzung genauer ausgearbeitet. Bereits in vorliegenden Untersuchungen zum Modell (Budden und Sagarin 2007; Gollwitzer und Sheeran 2006) fanden sich Hinweise, dass die Umsetzung der Handlungsabsichten verringert wird, wenn die Personen unter Stress stehen und die Handlungsfolgen für sie nicht vorhersehbar sind oder wenn sie starke Verhaltensgewohnheiten zu ändern versuchen (Adriaanse et al. 2011). In Abb. 1 wird ein erweitertes Rubikonmodell wiedergegeben, das die Planung der Umsetzung und die Unterstützung in der Umsetzungsphase beschreibt.

\section{A. Motivation und Ressourcenaktivierung}

Im ursprünglichen Rubikonmodell nach Heckhausen (1991) wird in der prädezisionalen motivationalen Phase lediglich das individuelle Abwägen der motivationalen Werte verschiedener Ziele betrachtet. Im erweiterten Modell werden dagegen zusätzlich die Ressourcen des Individuums und im sozialen und organisationalen Kontext berücksichtigt. Wie dies unten in zwei Bankprojekten näher beschrieben wird, ist es förderlich, wenn bei der Zielformulierung die organisationalen Vorgaben sowie die beteiligten Führungsebenen und das Team einbezogen wird und in individuelle Zielintentionen transformiert werden.

\section{B. Präaktionale Vorbereitung}

Im erweiterten Phasenmodell wird in Anlehnung an Storch (2004) die präaktionale volitionale Phase in zwei Teilphasen untergliedert. Die Phase B1 entspricht im Wesentlichen der Phase B im ursprünglichen Modell mit der Formulierung einer festen Umsetzungsabsicht, mit der die Person „ihren
Zugrundeliegende Prozesse

(A) Motivation und
Ressourcenaktivierung

(B1) Präaktionale Vorbereitung (Volition)

(B2) Präaktionale Vorbereitung (Planung der Ressourcen und ihrer Umsetzung)

(C) Aktionale Umsetzungund postaktionale Reflexion (Situation und Handlungen) und Remotivierung

(D) Postaktionale Bewertung und Reflexion nach dem gesamten Prozess und Zukunftsplanung
Phasen

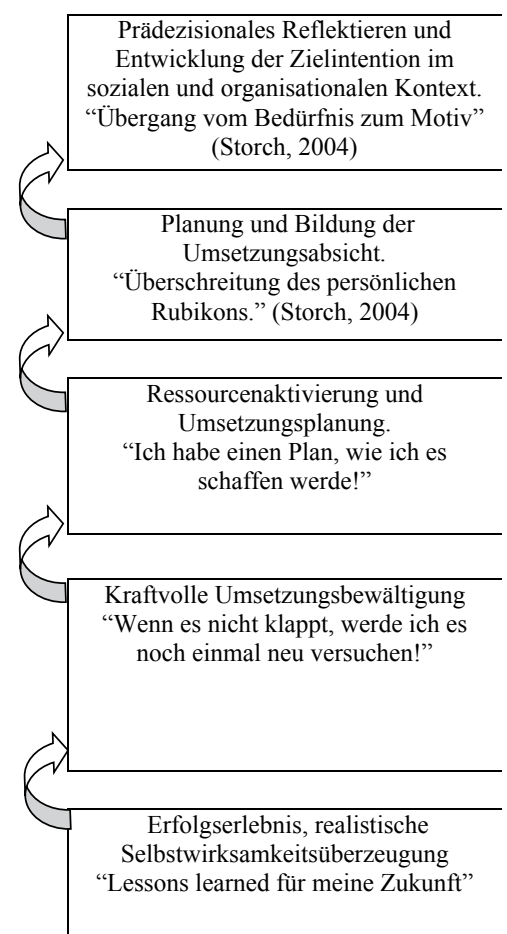

Abb. 1 Erweitertes Rubikonmodell (modifiziert nach Greif 2013, S. 133, mit Zitaten von Storch 2004 zu den Phasen A und B1)

persönlichen Rubikon überschreitet“". Storch (2004) ergänzt die Phase B2 zur Planung der individuellen Ressourcen bei der Umsetzung der Handlungsabsichten. Storch und Krause (2007, S. 132 f.) halten es beim Coaching und in der Psychotherapie für erforderlich, dass sich die Klient(inn)en auf die Schwierigkeiten in der Umsetzungssituation vorbereiten, insbesondere ob die Handlungsfolgen vorhersehbar oder nicht vorhersehbar sind. Für die Motivierung der späteren Umsetzung ist es wichtig, dass die Person überzeugt ist, dass sie einen bestmöglichen Plan entwickelt hat, der sie gegebenenfalls auch auf die Bewältigung unvorhersehbarer Handlungsfolgen vorbereitet (siehe die Maxime oben). Diese Überzeugung wird in Abb. 1 mit dem Satz ausgedrückt: „Ich habe eine Plan, wie ich es schaffen werde!“

In der Vorbereitungsphase ist immer auf potenzielle Änderungswiderstände im sozialen Umfeld zu achten. Wenn möglich sollen sie präventiv beeinflusst werden, bevor sie stark werden. Wie dies unten bei zwei Bankprojekten näher beschrieben wird, ist es dazu förderlich, den beteiligten Führungsebenen und dem Arbeitsteam die demotivierende Wirkung negativer Reaktionen auf individuelle Veränderungsversuche bewusst zu machen und sie anzuhalten, sich untereinander nicht destruktiv zu verhalten. Beginnend in der Phase A der Zielklärung sollen die individuellen Umsetzungsabsichten durch gemeinsames, verbindlich vereinbartes Commitment unterstützt werden. 


\section{Aktionale Umsetzung und postaktionale Reflexion}

Da sich in der Umsetzungsphase, wie oben beschrieben, Handlungen, Beobachtungen der Handlungsfolgen und postaktionale ergebnisorientierte Problem- und Selbstreflexionen sowie erneute Zielklärungen und Formulierungen von Umsetzungsabsichten und -plänen in schneller Folge zusammen zyklisch wiederholen können, werden im erweiterten Modell aktionale Umsetzung und postaktionale Reflexion nicht getrennt. Bei schwierigen Zielen und Umsetzungsabsichten (sowohl bei nicht vorhersehbaren Handlungsfolgen, als auch beim Ändern von Verhaltensgewohnheiten) ist es eine wichtige psychologische Funktion dieser Phase, die Motivation trotz eventueller Misserfolge oder frustrierend langsamer Veränderungen aufrechtzuerhalten oder die Person möglichst unmittelbar zu ,remotivieren“, wenn sie durch unerwünschte Ergebnisse frustriert wird und dazu tendiert aufzugeben. Dass dabei die anfänglich beschlossenen Lösungen trotz Schwierigkeiten nicht immer starr beibehalten, sondern situationsangemessen variiert erprobt werden können, wird mit dem Satz ausgedrückt: „Wenn es nicht klappt, werde ich es noch einmal neu versuchen!“

Geeignete Coachingmethoden für die Umsetzungsphase $\mathrm{C}$ sowie für die postaktionale Gesamtbewertung und Reflexion (Phase D) werden im Folgenden näher beschrieben.

\section{Coachingmethoden zur Umsetzungsunterstützung}

Die Umsetzung des Gelernten in die Praxis ist ein klassisches Thema der betrieblichen Aus- und Weiterbildung. So genannte Umsetzungs- oder Anwendungsverträge am Ende von Seminaren sind im Training sozialer Kompetenzen seit den 1970er Jahren bekannt (Semmer und Pfäfflin 1978, S. 178 f.). Eine Übersicht über verschiedene Konzepte zum Transfermanagement hat Lemke (1995) zusammengestellt. Das folgende Konzept zum Virtuellen Transfercoaching (Geißler 2011) knüpft an diese Methoden zur Förderung des Lerntransfers an.

\subsection{Virtuelles Transfercoaching}

Nach Harald Geißler (2011, S. 124) erfordert der Transfer des Gelernten in die Praxis nach Seminaren ein ,zusätzliches, neues Lernen“. Die Teilnehmenden müssen lernen, „im Arbeitsalltag bestimmte ,Rillen', langjährig eingeschliffene - vertraute und Sicherheit spendende - Gewohnheiten und Selbstverständlichkeiten zu verlassen, um neue - aus der Sicht der Organisation bessere Gewohnheiten und Selbstverständlichkeiten aufzubauen und ,einzuschleifen““ (ebd.). Er hat dazu ein Coachingformat entwickelt, das in der Ursprungsversion im Anschluss an Weiterbildungsse- minare den Lerntransfer sichern soll. Es kann auch auf ein virtuell unterstütztes Zielcoaching folgen.

Voraussetzung ist eine verbindliche Dreiecksvereinbarung zwischen den Vorgesetzten, den Teilnehmenden und den Trainer(inne)n. Geißler bevorzugt Einzelcoaching als Methode, weil dadurch individuell geklärt werden kann, ob die Teilnehmenden die angestrebten Veränderungen wollen. Außerdem braucht das ,zarte Pflänzchen“ des neu Gelernten im Alltag Schutz und Pflege und muss im Einzelfall an die Situation angepasst werden.

Einzelcoaching ist kostenintensiv. Geißler nutzt deshalb eine Kombination von Selbstcoaching mit einem Internetprogramm und Telefoncoaching. Mit Hilfe eines Internetprogramms beantworten die Teilnehmenden vorgegebene Fragen zum geplanten Transfer und geben Einschätzungen ab. Beispiele sind: „Wie zufrieden sind Sie auf einer Zehnerskala $(10=$ sehr zufrieden $)$ mit der Umsetzung Ihres Planes?“ „Was ist Ihr Ziel für den Zeitraum bis zu unserem nächsten Telefontermin?“ (Kreggenfeld und Reckert 2008, S. 221). Mit dem virtuellen Selbstcoaching-Tool werden Telefoncoachings vorbereitet. Die Verschriftlichung durch die Klient(inn)en erhöht die Verbindlichkeit und schafft einen Impuls zur gezielten Selbstreflexion, zwei Aspekte, die aller Erfahrung nach die Wirksamkeit von Coachings erhöhen. Dadurch kann die Länge der Telefonate kurz gehalten werden (maximal $30 \mathrm{~min}$ ). Im Anschluss an die Gespräche können die Teilnehmenden ihre Antworten im Internetprogramm korrigieren und dem Coach online Einsicht gewähren.

Kreggenfeld und Reckert (2008, S. 220 f.) verweisen als theoretische Grundlagen auf das klassische kybernetische Modell des zielgerichteten Handelns von Miller et al. (1974), für das präzise Nachfragen des Coachs im Telefoncoaching auf das Meta-Modell der Sprache von Bandler und Grinder (2001) und für die Fokussierung der Aufmerksamkeit auf Lösungen und konkrete Schritte auf die Lösungsorientierte Beratung nach de Shazer (1997). Das oben beschriebene erweiterte Rubikonmodell war bei der Veröffentlichung der Methode noch nicht publiziert. Wir ziehen es im vorliegenden Beitrag als allgemeines Phasenmodell heran und ordnen das virtuelle Zielcoaching als Methode der Phase der Motivations- und Zielklärung (Phase A) zu. Das virtuelle Transfercoaching dient dagegen schwerpunktmäßig, wie die Phase B2 des erweiterten Rubikonmodells, der Vorbereitung und Planung der Umsetzung und der Begleitung der Umsetzung (Phase C). Unberücksichtigt bleibt in Geißlers Methoden allerdings die für die Überschreitung des ,persönlichen Rubikons" psychologisch wichtige Formulierung der Handlungsabsichten (Phase B1). Denkbar wäre, dass hierzu ein weiteres virtuelles Modul konstruiert wird oder als Erweiterung in das virtuelle Zielcoaching aufgenommen wird. 
Geißler (2011, S. 132) schätzt, dass die Transferrate bei traditionellen Trainings nur bei $10-15 \%$ liegt. Nach seinen Beobachtungen werden mit drei VTCs in einem Zeitraum von 10-12 Wochen nach Einschätzungen der Teilnehmenden bemerkenswert hohe Tranferquoten von $89 \%$ erzielt. Da er allerdings soziale Erwünschtheit bei diesen Einschätzungen nicht ausschließt, vermutet er, dass die „objektive“ Transferrate nur 70-80\% erreicht. Weiter unten werden im vorliegenden Beitrag zwei Anwendungsbeispiele mit dem VTC vorgestellt, die zu bemerkenswerten wirtschaftlichen Ergebnissen geführt haben.

Wie oben zitiert, konzentriert sich Geißler (2011) inhaltlich vorwiegend auf Coaching zur Entwicklung neuer Verhaltensgewohnheiten. Um feste Verhaltensgewohnheiten zu verändern, wäre es erwägenswert, den Fokus stärker auf die Auslöser der Gewohnheit zu richten und die Umsetzung des veränderten Verhaltens über einen längeren Zeitraum mit dem VTC zu unterstützen. Die Methode ließe sich im Prinzip auch für das Managen komplexer Umsetzungssituationen mit unerwarteten Folgen der umgesetzten Handlungen adaptieren. Auch hier wären allerdings im Allgemeinen zusätzliche Zyklen der Zielklärung und des virtuellen Transfercoachings erforderlich.

\subsection{Telefon-Shadowing}

Telefon-Shadowing (TS) kann als Alternative oder Ergänzung zum Virtuellen Transfer Coaching bei schwierigen Umsetzungssituationen verwendet werden oder dort zum Einsatz kommen, wo Arbeitnehmergremien die Nutzung virtueller Medien zur Coachingunterstützung und/oder die Verschriftlichung untersagen. Die Methode basiert auf einer Kombination von Präsenz- (face-to-face) und Telefoncoaching. Der Begriff Shadowing kommt aus dem Englischen und bedeutet „Beschattung“. Im Sportcoaching ist eine Beobachtung und psychologische Begleitung der Sportler(innen) in der Umsetzungssituation selbstverständlich, im Coaching ist dies bisher eher selten, obwohl Shadowing nach vor liegenden Erkenntnissen außerordentlich wirksam ist (zusammenfassend siehe Greif 2013).

Im Prinzip ist es nicht neu, dass sich Coaches zwischen den Sitzungen telefonisch mit ihren Klient(inn)en in Verbindung setzen und mit ihnen Erfahrungen bei der Umsetzung ihrer Ziele besprechen. Das Besondere an der Methode ist, dass sie sich sehr genau am erweiterten Rubikonmodell orientiert, wie es oben in Abb. 1 wiedergegeben wird. Bevor das TS verwendet werden kann, wird in der Regel in faceto-face-Coachings nach dem erweiterten Rubikonmodell in Phase A eine Zielklärung durchgeführt, mit anschließender Formulierung der Umsetzungsabsicht in Phase B1 und Planung der Ressourcen in Phase B2 (Leittexte auf Anfrage bei Greif). Wie oben beschrieben, soll in der Planung der Umsetzung und Begleitung durch Coaching berücksichtigt werden, ob Verhaltensgewohnheiten verändert werden müssen und ob die Handlungsfolgen in der Umsetzungssituation vorhersehbar oder ungewiss sind (siehe auch Storch und Krause 2007, S. 132). TS mit mehrfachen postaktionalen ergebnisorientierten (Selbst)Reflexionen in der Umsetzung in Phase $\mathrm{C}$ erfolgen meist ebenfalls per Telefon.

Wenn in der Umsetzungsphase alles wie geplant und subjektiv erfolgreich verlief, kann die wertschätzende Anerkennung dieser Leistung durch den Coach die Umsetzungsmotivation weiter stärken. Wenn es nicht um eingeschliffene Gewohnheiten geht, genügen oft zwei bis vier kurze Telefonate. Wenn sich die Selbstbeobachtung und Umsetzung stabilisiert, genügen kurze Emails oder SMSNachrichten zur Unterstützung. Klient(in) und Coach „bleiben dran" an der Umsetzung.

Werden die Umsetzungsabsichten und/oder die Ziele nicht erreicht, kann eine Redefinition der Ziele und Umsetzungsabsichten erforderlich werden. Es ist wichtig, den Telefontermin möglichst kurz nach der geplanten Umsetzung zu vereinbaren. Der psychologische Vorteil des Telefoncoachings direkt nach der Situation ist, dass die oft starke Demotivierung durch das Misserfolgserlebnis schnell abgefangen werden kann. Eine „Remotivierung“ ist erfahrungsgemäß leichter, wenn sie sofort erfolgt, bevor sich Frustrationsgefühle resignativ verfestigt haben. Die Wahrscheinlichkeit ist groß, dass die Klient(inn)en ohne Unterstützung schnell innerlich aufgeben.

Im Verlauf des TS sollen sich die Coaches in der Umsetzungsunterstützung immer weiter zurücknehmen und die Eigeninitiative ihrer Klient(inn)en bei der ergebnisorientierten Reflexion und Umsetzung fördern. Am Ende in der Phase D zur postaktionalen Bewertung und Reflexion nach dem gesamten Prozess und Zukunftsplanung sollen in einem gemeinsamen face-to-face-Rückblick auf den gesamten Prozess die „Lessons learned“ der selbständig anwendbaren Methoden und Entwicklungspotenziale für künftige Herausforderungen herausgearbeitet und bekräftigt werden.

\subsubsection{Erfahrungen mit Telefon-Shadowing}

Greif hat als Berater und Coach bei organisationalen Veränderungsprozessen seit den 1980er Jahren zuerst mit Faceto-face-Shadowing, später vorwiegend mit TS sehr positive Erfahrungen gemacht. Bei intrinsisch motivierten, wichtigen Verhaltensänderungen können nach seinen Erfahrungen und denen von über 20 anderen Coaches erfahrungsgemäß eine objektivierbare Umsetzungsraten von über $90 \%$ erreicht werden. Die Wirksamkeit zeigt sich besonders bei der Veränderung eingefahrener Gewohnheiten, die durch übliche Coachingmethoden nicht oder nicht nachhaltig beeinflusst werden können.

Der zeitliche Aufwand bei der Verwendung von TS ist höher als beim Virtuellen Transfercoaching. Um den 
Aufwand zu verringern, werden in einem laufenden, mit EUREGIO-Mitteln geförderten Projekt, Cloud- und Smartphone-Tools zur Unterstützung der Zielklärung, Formulierung von Umsetzungsabsichten und zum TS entwickelt (Anfragen an Greif). Die Tools sollen Coaches nicht ersetzen. Die Zielklärung und die Formulierung der Handlungsabsicht sollen face-to-face, das erste Shadowing-Gespräch und bei Bedarf auch weitere sollen per Telefon durchgeführt werden. Die Smartphone-Tools sollen die Klient(inn) en zusätzlich dazu anhalten, die Ziele im Auge zu behalten und sie an die geplante Umsetzung der Handlungsabsichten erinnern.

\section{Praxis und Forschung}

\subsection{Ausgangssituation vieler Coaching Projekte}

Sich neu und anders zu verhalten ist im Beruf oft keine freiwillige Entscheidung. Anforderungen an Verhaltensveränderungen tauchen in Form von neuen Managementvorgaben und veränderter Regulatorik auf oder ergeben sich aus organisationalen und technologischen Veränderungen. So werden zum Beispiel neue Tools eingeführt, Prozesse geändert oder eine neue Haltung am Kunden und gegenüber Mitarbeiter(inne)n gefordert. Viele dieser Veränderungen erfordern eine Änderung bestehender Verhaltensroutinen der ausführenden Mitarbeiter(inn)en und Führungskräfte. Meistens wird die Veränderungstiefe vom Top Management unterschätzt und Veränderungsprozesse werden nicht professionell erfasst und begleitet. In diesen Fällen beobachten wir häufig Störfaktoren und Widerstände, die sich beispielsweise in einem Abfall der Produktivität, ineffektiven Workarounds oder schlicht in der Ignoranz des neu Geforderten äußern. Jedoch auch gängige Qualifikationsangebote wie Webinare oder Seminare und Trainings stoßen schnell an Grenzen, wenn es um den Aufbau neuer Verhaltensroutinen geht. Das vielerorts geforderte Vorleben durch die Führungskraft verändert so wenig wie die kaskadische Kommunikation des Neuen. Was also tun, wenn viele ihr Verhalten verändern sollen? Wie entsteht Motivation für das Neue in der Breite? Wie formt sich aus dem Action Intention Gap ein Umsetzungswille jedes einzelnen? Wie ersetzt sich eine alte Gewohnheit durch eine neue Verhaltensroutine? Die Beantwortung dieser Fragen ist zentral für das Gelingen von Veränderungen von Individuen, Teams und Organisationen.

\subsection{Zwei Beispiele einer ganzheitlichen}

Veränderungsbegleitung mit ergebnisorientiertem Coaching

Zwei Projekte aus der Praxis einer Unternehmensberatung seien hier exemplarisch vorgestellt. In beiden wurde von Mitarbeiter(inn)en und Führungskräften ein Verhalten erwartet, das neu und außerhalb der bestehenden Routinen und Komfortzonen lag. Im ersten Beispiel sollten etwa 80 Kundenberater(innen) vermögende Kund(inn)en, die bisher weitgehend unentgeltlich betreut wurden, überzeugen, dass sie einen mit Kosten verbundenen Vertrag mit der Bank abschließen. In dem zweiten Beispiel galt es in einer Großbank mit mehreren tausend Berater(inne)n und Führungskräften, die Ansprache der Kund(inn)en sowie die Anwendung eines neuen Beratungstools bis zum Abschluss $\mathrm{zu}$ aktivieren und zu verbessern. Höhere Kundenzufriedenheit, mehr Wachstum und mehr Profitabilität waren die damit einhergehenden Ergebniserwartungen des Auftraggebers.

In beiden Fällen wurde gemeinsam mit zeb, einem der größten Beratungsunternehmen im Finanzdienstleistungssektor, ein Vorgehen gewählt, das besondere Berücksichtigung der in diesem Artikel erörterten Förderung der konkreter Umsetzungsabsichten (Volition) und der Umsetzungsbegleitung einräumt. Darüber hinaus unterscheidet es sich in wesentlichen Punkten von der herkömmlichen Praxis der Change- und Qualifikationsarchitekturen im Finanzdienstleistungsbereich.

1. Statt in Off-the-Job-Seminaren einzelne zu befähigen, gehen wir vor Ort in die Niederlassungen oder Filialen und arbeiten dort mit der Führungskraft und ihrem Team on the job und am Kunden. So finden organisationale und situative Kontexte Berücksichtigung und Individualziele können im Team und in der Hierarchie in Einklang gebracht werden. Zudem konzentriert sich alles auf den wesentlichen Punkt der Wertschöpfung nämlich der Arbeit am und mit dem Kunden.

2. Statt Vorgaben des Managements umzusetzen, bestimmt jedes Team und jede(r) Mitarbeiter(in) seine/ihre eigene Selbstentwicklung im Rahmen der vorgegebenen Unternehmensziele. Diese wird von der Einwilligung und Absichtserklärung zur konkreten Umsetzungsabsicht transformiert.

3. Statt einer Einmalintervention implementieren wir einen systematischen Prozess angelehnt an das ergebnisorientierte Coaching (Greif 2008). Das Vorgehen erlaubt den Prozess von der Problemreflexion über Selbsteinschätzungen und individuelle Zielsetzungen sowie Formulierung individueller Umsetzungsabsichten nach dem Rubikonmodell bis zur Verstetigung neuer Verhaltensroutinen für eine sehr große Anzahl von Mitarbeiter(inn) en und Führungskräften möglich zu machen. Die Führungskräfte sind anschließend in der Lage, den Prozess in ihrer Linienfunktion weiter zu begleiten. Virtuelle Coaching-Formate (Geißler 2011, 2008, S. 220 f.) und ein neues Führungsinstrument unterstützen eine kontinuierliche Verbesserung. Sie helfen, die Veränderungsabsichten konsequent umzusetzen, neue Gewohnheiten 
zu entwickeln und unvorhergesehene Schwierigkeiten zu bewältigen (s. o.).

\subsubsection{Von der Selbstreflexion zur Volition}

Unser Vorgehen beginnt entsprechend dem ergebnisorientierten Coachings mit dem Aktivieren von Problem- und Selbstreflexionen als wesentlichem Ausgangspunkt der bewussten Selbstveränderung. Bei den hier genannten Beispielen erfolgte dies durch eine Stärken-Schwächen-Einschätzung des Einzelnen und des Teams in einem Gespräch zum derzeitigen Vertriebserfolg zwischen dem zeb Business-Coach und jedem Beteiligten. Damit erreichen wir eine richtunggebende Orientierung für die zu behandelnden Probleme aus der Sicht jedes Einzelnen. Da jede(r) Mitarbeiter(in), deren Führungskraft sowie deren Vorgesetzte eine Einschätzung vornehmen, ergeben sich auch rollenbedingt unterschiedliche Stärken- und Problemwahrnehmungen. Diese Phase der Problem- und Selbstreflexion kann durch den Einbezug der antizipierten gegenseitigen Rückmeldungen und angenommener Rückmeldungen der Kunden weiter verstärkt werden und eine selbstkritischere Haltung der Befragten fördern (Benning-Rohnke und Greif 2010). Die individuellen Einschätzungen werden in einem folgenden Workshop mit allen Beteiligten offengelegt. Mit der Sichtbarmachung von Uneinigkeit entstehen an dieser Stelle zunächst oft Konflikte. Vor allem Einschätzungen, die von der eigenen abweichen, lösen oft negative Emotionen (Ungerechtigkeitsgefühle, Ärger, Enttäuschung) aus, die sich sowohl gegen die Führungskraft als auch gegen Teammitglieder richten können. Unsere Erfahrungen bestätigen dennoch eine insgesamt förderliche Wirkung einer frühen Offenlegung solcher nach Larson et al. (1998), ,ungeteilten Informationen“. Die Reflexionen über Stärken und Schwächen aller Beteiligten werden dadurch erweitert und tragen zur Erhöhung der Qualität der Lösungen bei. Die kognitiven und emotionalen Prozesse gilt es jetzt zum Konsens zusammenzuführen und auf ein gemeinsames Veränderungsziel hin auszurichten. Das ist die Aufgabe des externen Business-Coachs, der den Prozess lösungsoffen zu einer Zielintention moderieren muss, hinter der sich alle Beteiligte motiviert versammeln können. Der Business-Coach sichert dabei gleichzeitig den Bezug des Teamziels zu den vorgegebenen Unternehmenszielen und eine Zielformulierung nach den SMART-Kriterien (Doran 1981). Der Konsens im Team ist wesentlich für den nachfolgenden Veränderungserfolg. Denn ein Dissens zwischen den Veränderungswünschen der Führungskraft und dem Team bzw. einzelnen Mitarbeiter(inne)n ist in der Praxis eine gängige, jedoch oft unterschätzte und unbeachtete, Störquelle in der Umsetzung der Qualifikations- und Change-Maßnahmen. Der Aufwand bis zur gemeinsam geteilten Zielintention scheint hoch, doch durch diesen Prozess einer maximalen personalen Partizipation aller Beteiligten kann bereits eine hohe Akzeptanz und nachhaltige Identifikation mit der Zielintention erreicht werden.

Als Bild nutzen wir das einer „Erfolgstreppe“, mit der die für den Erfolg notwendigen Stufen von der Ausgangsituation, über den Gruppenkonsens mit verbindlichen gemeinsamen Zielen, bis zu den Zielen und Umsetzungsabsichten der Einzelnen veranschaulicht werden. Anhand dieses Bilds initiieren wir die Diskussionen, Zielklärungen und Formulierungen der konkreten Umsetzungsabsichten. Aus der Beobachtung und der Rückmeldung von Teilnehmer(inn) en können wir feststellen, dass die Intensität und die Qualität der Teamenergie durch diesen Prozess mit den Stufen der „Erfolgstreppe“ bereits deutlich positiv beeinflusst und erste Verhaltensänderungen aktiviert werden. Selbst vorher eher passive Mitarbeiter(innen) oder „Querköpfe“ bringen sich nach unseren Beobachtungen plötzlich wieder in die Gruppe ein und werden nicht selten Treiber der Umsetzung. Nach Bruch und Vogel (2005) sind Intensität und Qualität der Energie ausschlaggebende Faktoren für die Erreichung der Unternehmensziele. Im Fall von Verhaltensänderungen ist dies nach unseren Beobachtungen eine notwendige jedoch nicht hinreichende Bedingung.

Für die Veränderung routinierter Verhaltensweisen bedarf es individueller Selbstentwicklungsziele und Umsetzungsabsichten eines jeden einzelnen Teammitglieds. Die gemeinsame Motivation und Zieldefinition des Teams muss, wie oben im Rubikonmodell beschrieben, in bewusste Willensentscheidung jedes Einzelnen transformiert werden, sich konkret und konsequent anders zu verhalten, um die gemeinsam formulierte Zielintention zu realisieren. Zur Formulierung des individuellen Selbstentwicklungsziels nutzen wir wiederum das Bild der Erfolgstreppe und lassen, methodisch ähnlich wie in den gängigen Untersuchungen (Gollwitzer und Sheeran 2006), die konkreten Umsetzungsabsichten in „Stillarbeit" von jedem einzelnen in erneuter Selbstreflexion entwickeln. Dadurch wird die Zielintention mit individueller Volition unterlegt. Die Voraussetzungen für praktische Handlungsoptimierungen sind geschaffen. Klassische Qualifikationsansätze berücksichtigen die bisher aufgezeigten Schritte in der Regel nicht und wir vermuten, dass dort das Fehlen von Freiwilligkeit, Teamkonsens und individueller Volition wesentliche Gründe für die geringe Transferquote traditioneller Trainings und Qualifikationsmaßnahmen ist.

\subsubsection{Neues Verhalten aufbauen und verstetigen}

Die individuelle Erfolgstreppe mit ihren Stufen bildet die Basis für ein on the job begleitendes ergebnisorientiertes Präsenzcoaching, das je nach individueller Zieldefinition 
mit Training- und Consultingelementen angereichert werden kann um das Selbstentwicklungsziel zu erreichen.

Die Mitarbeiter(innen) und Führungskräfte erleben in der Regel sehr schnell einen Erfolg. In der Praxis beobachten wir, dass Mitarbeiter(inn)en durch ihre Erfolgserlebnisse so sehr motiviert sind, dass sie beginnen, sich weitere Ziele zu setzen und anschließend selbst weitere Erfolgstreppen zu formulieren. Gelingt es jetzt den Vorgesetzten, die begonnen Veränderungen mit den im Unternehmen verfügbaren Instrumenten und Formaten zu begleiten, werden die Verhaltensverbesserungen des Einzelnen verstetigt und in Summe hat das Team eine Chance, gemeinsam kontinuierliche selbstgesteuerte Verbesserungen umzusetzen.

Neue Verhaltensweisen scheitern oft an kleinen Hürden und Banalitäten. Es besteht die Gefahr des Rückfalls in alte Gewohnheiten. Durch den von Geißler (2011) entwickelten virtuellen Coaching Ansatz oder ein gut ausgeführtes Telefon-Shadowing wird möglichen Rückfällen entgegengewirkt. Nach unserer Einschätzung sind diese die Führungsarbeit begleitende Maßnahmen unerlässlich für den nachhaltigen Aufbau neuer Routinen. Eine konsequente Begleitung im Anschluss an die Präsenzphase mit diesen Methoden stimuliert Mitarbeiter(innen) und Führungskraft gleichermaßen, im Trubel des operativen Geschäfts, den Fokus auf das Ziel und die notwendigen Veränderungen zu behalten und Stolpersteine aus dem Weg zu räumen. Es überrascht, wie selten so kostengünstige wie effektive Maßnahmen im betrieblichen Umfeld bisher Anwendung finden. Viele Investitionen in Mitarbeiter(inn)en verpuffen ohne nachhaltige Wirkungen.

\subsubsection{Die Ergebnisse}

Das hier skizzierte ganzheitliche Vorgehen von der Problem- und Selbstreflexion über die Zielintention zur Umsetzungsabsicht bis zum Aufbau und zur Verstetigung des neuen Verhaltens wurde in beiden Anwendungen im Bankenumfeld evaluiert.

Beispielhaft seien die Verbesserungen in den Key Performance Indices (KPI) eines Panels genannt. Die Zielerreichung bei der Profitabilität hat sich von vorher 92 auf nachher $118 \%$ um $29 \%$ verbessert, der Kundenzuwachs sogar um $105 \%$ von 38 auf $78 \%$, die Qualität der Dienstleistung auf hohem Niveau immerhin um 3\% von 128 auf $132 \%$. Viele Führungskräfte kommentierten spontan, dass dieses Programm mit ergebnisorientiertem Coaching erstmals unmittelbare und große Veränderungen im Unternehmen bewirkt hat. Die Maßnahme gilt in der Bank unter allen Stakeholdern als die beste, jemals durchgeführte Qualifizierungsmaßnahme. Anhand der vorliegenden Ergebnisse der Evaluation können wir nicht feststellen, welchen Anteil am Erfolg des gesamten Programms das Coaching oder andere einzelne Elemente haben. Nach den spontanen Rückmel- dungen der Beteiligten sind die Erfolgstreppe als bildhafte Konkretisierung des Umsetzungswillens und das Transfercoaching zentrale und für viele Veränderungserfolge unerlässliche Elemente.

\section{Perspektiven}

Große praktische und wissenschaftliche Herausforderungen wie die Überbrückung der Kluft zwischen Intentionen und Handlungen erfordern eine intensive Zusammenarbeit von Forschung und Praxis. Wir können hoffen, dass das Interesse von Coaches zunimmt, wissenschaftlich fundierte und praktisch innovative Methoden wie das Virtuelle Transfercoaching oder die Telefon-Shadowing-Methode in weiteren Studien auszuprobieren und ihre Wirksamkeit zu überprüfen. In einer Pilotstudie mit 14 professionellen Coaches (Leder 2013) erreichte die Bereitschaft, die Telefon-Shadowing-Methode anderen Coaches zu empfehlen, auf einer Skala von 0-10 den außerordentlich hohen Wert von 9,18. Im oben geschilderten Bankprojekt waren die Führungskräfte, mit einem ähnlich hohen Wert von 9,0 bereit, die Trainer(innen) und Coaches weiterzuempfehlen.

Um wissenschaftliche Erkenntnisse praktisch nutzbar zu machen, sind intensive Diskussionen und Kooperationen von Wissenschaft und Praxis erforderlich. Für diese Zusammenarbeit steht auch, dass der vorliegende Beitrag von einem Wissenschaftler geschrieben wurde, der als Coach praktisch arbeitet und einer Unternehmensberaterin mit Psychologie- und Coachingexpertise, die mit ihren Anforderungen und Beobachtungen den Wissenschaftler vorantreibt, wissenschaftliche Erkenntnisse zu nutzen, um bessere Methoden zu entwickeln. Wie hier am Beispiel der Forschung zum Rubikonmodell und zur Veränderung von Verhaltensgewohnheiten gezeigt wurde, ist es in der Zusammenarbeit erforderlich, die in der Wissenschaft eingesetzten Methoden auf der Basis von Erfahrungswissen für die praktische Anwendung anzupassen und in umfassende Interventionen zu integrieren. Die Anwendungsbeispiele im Bankenbereich stehen für eine souveräne Adaptation und Nutzung wissenschaftlicher Konzepte und Methoden bei großen und komplexen organisationalen Changes im Verbund mit Change Management, Training und Umsetzungsvereinbarungsgesprächen mit Niederlassungsleitern.

Damit Handlungsabsichten umgesetzt werden und die angestrebten Ziele erreicht werden, genügt es anscheinend nicht, wie im ursprünglichen Rubikonmodell von Heckhausen (1991), lediglich die individuellen Ziele zu klären und Willensentscheidungen zu treffen. Nach unseren Beobachtungen und Felderfahrungen kommt es entscheidend auf eine gemeinsame Zielklärung und Commitment zur individuellen Umsetzungsunterstützung im gesamten organisationalen Umfeld an. Auch in der Umsetzungsphase spielt eine unter- 
stützende Begleitung durch die höheren Führungsebenen und das Team eine große Rolle neben dem Coaching. Im erweiterten Rubikonmodell wird deshalb die Bedeutung des organisationalen Kontexts in allen Phasen besonders betont.

Wie unser Beitrag zu zeigen versucht, geht es in der Zusammenarbeit von Praxis und Wissenschaft in der zukünftigen Coachingforschung nicht nur um die Evaluation der Wirksamkeit von Methoden. Theorien und anwendungsorientierte Forschungsergebnisse der Wissenschaft eignen sich auch als Wissensgrundlage für einen Austausch zwischen Wissenschaft und Praxis über praktische Beobachtungen. Dadurch können, wie der vorliegende Beitrag exemplarisch zeigt, Theorien und Methoden praxisorientiert weiterentwickelt werden. Wir verstehen praxisorientierte wissenschaftliche Theorien als sozial konstruierte Denkwerkzeuge, die uns helfen, uns über komplexe praktische Fragestellungen mit anderen systematisch auszutauschen und zum Nutzen der Beteiligten innovative Veränderungen $\mathrm{zu}$ initiieren. Es ist eine faszinierende Vision, Erkenntnisse und Methoden aus weiteren Feldern der Grundlagenforschung zur Innovation der Coachingpraxis zu nutzen.

Open Access Dieser Artikel wird unter der Creative Commons Namensnennung 4.0 International Lizenz (http://creativecommons.org/ licenses/by/4.0/deed.de) veröffentlicht, welche die uneingeschränkte Nutzung, Verbreitung und Wiedergabe für beliebige Zwecke erlaubt, sofern Sie den/die ursprünglichen Autor(en) und die Quelle ordnungsgemäß nennen, einen Link zur Creative Commons Lizenz beifügen und angeben, ob Änderungen vorgenommen wurden.

\section{Literatur}

Adriaanse, M. A., Gollwitzer, P. M., De Ridder, D. T. D., de Wit, J. B. F., \& Kroese, F. M. (2011). Breaking habits with implementation intentions: A test of underlying processes. Personality and Social Psychology Bulletin, 37(4), 502-513.

Bandler, R., \& Grinder, J. (2001). Metasprache und Psychotherapie (3 Bde). Paderborn: Junfermann.

Benning-Rohnke, E., \& Greif, S. (2010). Kundenorientierung Warum sie oft scheitert und wie sie besser machbar ist. In G. Greve \& E. Benning-Rohnke (Hrsg.), Kundenorientierte Unternehmensführung. Konzept und Anwendung des Net Promoter Score in der Praxis (S. 117-156). Stuttgart: Gabler.

Bruch, H., \& Vogel, B. (2005). Organisationale Energie: Wie Sie das Potenzial Ihres Unternehmens ausschöpfen (uniscope. Die SGOStiftung für praxisnahe Managementforschung). Wiesbaden: Gabler.

Budden, J. S., \& Sagarin, B. J. (2007). Implementation intentions, occupational stress, and the exercise intention-behavior relationship. Journal of Occupational Health Psychology, 12(4), 391-401.

David, S., Clutterbuck, D., \& Megginson, D. (Hrsg.). (2013). Beyond goals: Effective strategies for coaching and mentoring. Farnham: Gower.

Doran, G. T. (1981). There's a S.M.A.R.T. way to write management's goals and objectives. Management Review, 70(11), 35-36.

Duhigg, C. (2012). The power of habit: Why we do what we do in life and business. London: Random House, Kindle.

Geißler, H. (Hrsg.). (2008). E-Coaching. Hohengehre: Schneider.
Geißler, H. (2011). Coaching meets Training - zur Lösung des Transferproblems durch ,virtuelles Transfercoaching (VTC)“'. In M. Loebbert \& R. Wegener (Hrsg.), Coaching entwickeln. Forschung und Praxis im Dialog (S. 123-134). Göttingen: Vandenhoeck \& Ruprecht.

Gollwitzer, P. M., \& Sheeran, P. (2006). Implementation intentions and goal achievement: A meta-analysis of effects and processes. $\mathrm{Ad}$ vances in Experimental Social Psychology, 38, 69-120.

Gollwitzer, P. M., Kuhl, J., \& Heckhausen, H. (1996). Das Rubikonmodell der Handlungsphasen. In J. Kuhl \& H. Heckhausen (Hrsg.), Enzyklopädie der Psychologie, Bd. 4, Motivation, Volition und Handlung (S. 531-582). Göttingen: Hogrefe.

Greif, S. (2008). Coaching und ergebnisorientierte Selbstreflexion. Göttingen: Hogrefe.

Greif, S. (2013). Putting goals to work in coaching: The complexities of implementation. In S. David, D. Clutterbuck, \& D. Megginson (Hrsg.), Beyond goals: Effective Strategies for Coaching and Mentoring (S. 125-149). Farnham: Gower.

Greif, S., Runde, B., \& Seeberg, I. (2004). Erfolge und Misserfolge beim Change Management. Göttingen: Hogrefe.

Heckhausen, H. (1991). Motivation and action. New York: Springer.

Kreggenfeld, U., \& Reckert, H.-W. (2008). ,Virtuelles Transfercoaching'. Die Transferquote verdreifachen. In H. Geißler (Hrsg.), E-Coaching (S. 217-224). Hohengehre: Schneider.

Lally, P., Jaarsveld, C. H. M., Potts, H. W. W., \& Wardle, J. (2010). How are habits formed: Modelling habit formation in the real world. European Journal of Social Psychology, 40(6), 998-1009.

Larson, J. R., Foster-Fishman, P. G., \& Franz, T. M. (1998). Leadership styles and the discussion of shared and unshared information in decision making groups. Personality and Social Psychology Bulletin, 24, 563-585.

Leder, S. (2013). Telefon-Shadowing: Wirksamkeit einer neuen Coaching-Methode zur Umsetzung von Verhaltensänderungen. Diplomarbeit, Bielefeld: Universität Bielefeld Arbeitseinheit Arbeits- und Organisationspsychologie.

Lemke, S. G. (1995). Transfermanagement. Göttingen: Verlag für Angewandte Psychologie.

Locke, E. A., \& Latham, G. P. (2002). Building a practically useful theory of goal setting and task motivation. American Psychologist, 57(9), 705-717.

Miller, G. A., Galanter, E., \& Pribram, K. H. (1974). Strategien des Handelns. Pläne und Strukturen des Verhaltens. Stuttgart: Klett.

Orbell, S., \& Verplanken, B. (2010). The automatic component of habit in health behavior: Habit as cue-contingent automaticity. Health Psychology, 29(4), 374-383.

Schwarzer, R. (2004). Psychologie des Gesundheitsverhaltens. Göttingen: Hogrefe.

Semmer, N., \& Pfäfflin, M. (1978). Interaktionstraining. Weinheim. Beltz.

Shazer, S. de (1997). Der Dreh: Überraschende Wendungen und Lösungen in der Kurzzeittherapie. Heidelberg: Carl Auer.

Sheeran, P. (2002). Intention-behavior relations: A conceptual and empirical review. In W. Stroebe \& M. Hewstone (Hrsg.), European review of social psychology (Bd. 12, S. 1-30). New York: Wiley.

Storch, M. (2004). Resource-activating selfmanagement with the Zurich Resource Model (ZRM). European Psychotherapy, 5(1), 29-66.

Storch, M., \& Krause, F. (2007). Selbstmanagement - ressourcenorientiert. Grundlagen und Trainingsmanual für die Arbeit mit dem Zürcher Ressourcen Modell ZRM (4. Aufl.). Bern: Huber.

Tierney, S., Mamas, M., Skelton, D., Woods, S., Rutter, M. K., Gibson, M..et al (2011). What can we learn from patients with heart failure about exercise adherence? A systematic review of qualitative papers. Health Psychology, 30(4), 401-410.

Yin, H. H., \& Knowlton, B. J. (2006). The role of the basal ganglia in habit formation. Nature Reviews Neuroscience, 7(6), 464-476. 